\title{
Management of postdural puncture headache with epidural blood patch in a child
}

\author{
Dong Ha Lee, and Eun-Ju Kim \\ Department of Anesthesiology and Pain Medicine, Daegu Fatima Hospital, Daegu, Korea
}

Postdural puncture headache (PDPH) is a common complication in adults for spinal anesthesia or epidural anesthesia but is rare in children because their normal cerebrospinal pressure is lower than in adults and the diagnosis of PDPH usually depends on a medical history. We report a case where a child was suspected of PDPH and was treated successfully with an epidural blood patch (EBP).

A 6-year-old boy at $110 \mathrm{~cm}$ and $16 \mathrm{~kg}$ came to the hospital complaining of postural headache. He had a diagnostic dural puncture done 3 days prior to his visit and began to complain of postural headache, dizziness, and nausea 2 days prior to his visit. Under the premise of $\mathrm{PDPH}$, conservative treatment such as bed rest and fluid therapy were done but the headache continued with accompanying vomiting. In the physical examination, the headache occurred when sitting or standing and slowly eased when he lay down. There were no other neurological abnormalities. EBP was done for diagnostic and treatment purposes under sedation using ketamine while monitoring oxygen saturation, ECG, and blood pressure. The loss of resistance technique was used with a 22-gauge Tuohy needle on L4-5. After confirming that the needle had entered into the epidural space approximately $13 \mathrm{~mm}$ from the skin, 2 $\mathrm{ml}$ of autologous blood as injected. It was decided to observe the patient's condition after he woke up and stabilized. After 12 hours of sufficient fluid therapy and bed rest, the postural headache, dizziness, and nausea all disappeared.

PDPH occurs 1-3 days after dural puncture and it can be accompanied by characteristic symptoms of postural headache, nausea, vomiting, and dizziness. When diagnosed with PDPH, conservative treatment such as bed rest, fluid therapy, and painkillers are prescribed at first, but when the symptoms do not improve after 24-48 hours of conservative treatment, a more aggressive treatment such as EBP can be done. EBP is not necessary for all PDPH patients, but in this case, the patient showed no improvement in his symptoms after 2 days of conservative treatment and showed severe PDPH symptoms of vomiting; therefore, EBP was done.

DiGiovanni and Dunbar [1] explained the easing of a headache with EBP in two ways. The initial rapid easing of the headache is the effect of the injected autologous blood creating a hematoma to pressure the dural and increase the pressure in the subarachnoid space. 7 hours later, this coagulation starts to dissolve and attaches to the dural as a form of thin membrane, which seals the CSF leaking through the build-up of organized clotted blood. The subsequent continuous production of CSF restores the pressure back to equilibrium. When doing an EBP, slow injection is necessary to feel for any pressure on the nerve roots, and injection must be stopped when there are clinical symptoms from pressure on the nerve roots.

In our patient, the volume effect was difficult to judge due to the ketamine induced sedated state, and 12 hours later, when the sedative effect had worn off, the symptoms for PDPH had disappeared. EBP in a sedative state is easy to do and can ease the anxiety of the patient. However, there could be delays in judging the effect of the EBP after the procedure and in recognizing the occurrence of complications such as pressure on the nerve root.

Children have a very thin epidural depth and small epidural

Corresponding author: Eun-Ju Kim, M.D., Department of Anesthesiology and Pain Medicine, Daegu Fatima Hospital, 576-31, Sinam-dong, Dong-gu, Daegu 701-600, Korea. Tel: 82-53-940-7434, Fax: 82-53-954-7417, E-mail: kej1127@fatima.or.kr

(c) This is an open-access article distributed under the terms of the Creative Commons Attribution Non-Commercial License (http:// creativecommons.org/licenses/by-nc/3.0/), which permits unrestricted non-commercial use, distribution, and reproduction in any medium, provided the original work is properly cited. 
space compared to adults. Therefore, the anesthesiologist must keep in mind the possibility of re-puncture of the dural due to the EBP and predict the depth of the epidural space before doing the EBP. Generally, the epidural depth of children from 6 months to 10 years of age is known to be $1 \mathrm{~mm} / \mathrm{kg}$ [2]. Choi et al. [3] reported in a study on lumbar epidural depth of Korean infants and children that the epidural depth ( $\mathrm{mm}$ ) was $9+0.5 \times$ weight $(\mathrm{kg})-0.02 \times$ age (months). Our patient's epidural depth was approximately $13 \mathrm{~mm}$, which was slightly thinner than the $15.34 \mathrm{~mm}$ reported by Choi et al. [3].

Ylönen and Kokki [4] reported that they did EBP on 7 children under the age of 12 with $0.13-0.46 \mathrm{ml} / \mathrm{kg}$ of blood and that the suitable amount of blood was $0.2-0.3 \mathrm{ml} / \mathrm{kg}$. In this case, a single EBP procedure with $2 \mathrm{ml}$ of blood was successful in relieving all symptoms of PDPH. The $2 \mathrm{ml}$ of blood was a smaller amount than the suitable amount (3.4-5.1 ml/kg) reported by Ylönen and Kokki [4]; however, these formulas were drawn from children from western countries so there could be differences in Korean children. In lumbar epidural anesthesia of children over $10,0.1 \times$ age $(\mathrm{ml})$ is needed to block one spinal segment [2], and $2 \mathrm{ml}$ is the volume that occupies approximately three spinal segments in 6-year-old children. If the blood is accurately inserted in the punctured area, $2 \mathrm{ml}$ of blood is thought to be enough to have a sufficient effect. When there is no relief of symptoms using conservative treatments such as bed rest and fluid therapy, EBP can be considered even in children with PDPH.

\section{References}

1. DiGiovanni AJ, Dunbar BS. Epidural injections of autologous blood for postlumbar-puncture headache. Anesth Analg 1970; 49: 268-71.

2. Dalens BJ. Regional anesthesia in children. In: Miller's anesthesia. 7th ed. Edited by Miller RD, Eriksson LI, Fleisher LA, WienerKronish JP, Young WL: Philadelphia, Elsevier Churchill Livingstone. 2010, pp 2534-5.

3. Choi JB, Hong JY, Kim JS, Kil HK. Estimating lumbar epidural space depth in infants and children of Korea. Korean J Anesthesiol 2009; 56: 531-4.

4. Ylönen P, Kokki H. Management of postdural puncture headache with epidural blood patch in children. Paediatr Anaesth 2002; 12 : 526-9. 